\title{
Hybrid Dyadic - MPIE Integral Equation Analysis of Passive Microwave Devices in Layered Media
}

\author{
Mark Vrancken, Guy A. E. Vandenbosch \\ Katholieke Universiteit Leuven \\ Department of Electrical Engineering \\ Division ESAT-TELEMIC \\ Kasteelpark Arenberg 10 \\ B-3001 Heverlee (Leuven), BELGIUM \\ phone $+32 /(0) 16 / 321820$ \\ fax $+32 /(0) 16 / 321986$ \\ Mark.Vrancken@esat.kuleuven.ac.be
}

\section{abstract}

Integral equation modeling of electromagnetic radiation and interference phenomena in planar circuits and antennas offers exact solutions usually at the cost of excessive computation time, especially when arbitrary 3D conducting surfaces are involved. However, we will restrict the geometry to the $2.5 \mathrm{D}$ case : this assumes that only horizontal current sheets parallel to the layer structure and vertical conducting surfaces normal to the dielectric layers are allowed. This case does nevertheless cover most of the practical problems. The limitation to the $2.5 \mathrm{D}$ case allows to perform more analytical work. A hybrid dyadic-mixed potential representation of the field is proposed as a viable alternative to the traditional 'total' mixed potential representations. Several examples show the validity of the proposed approach.

\section{THE 2.5D STRUCTURE IN THE LAYERED MEDIUM}

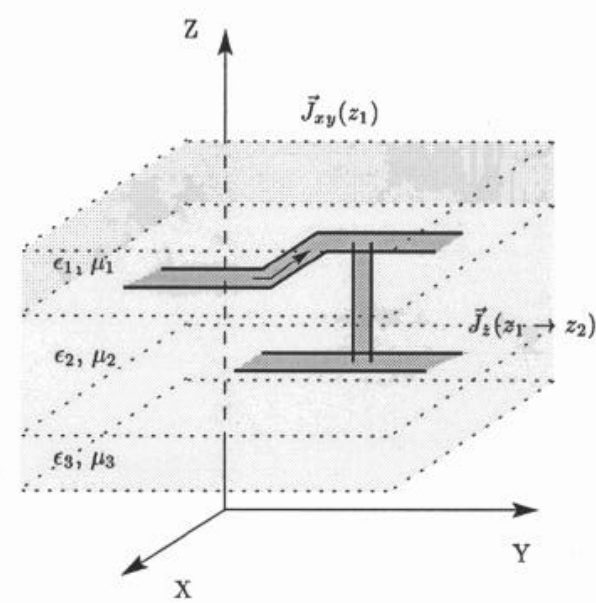

Figure 1: The usual $2.5 \mathrm{D}$ or $3 \mathrm{D}$ planar structure in a layered medium.

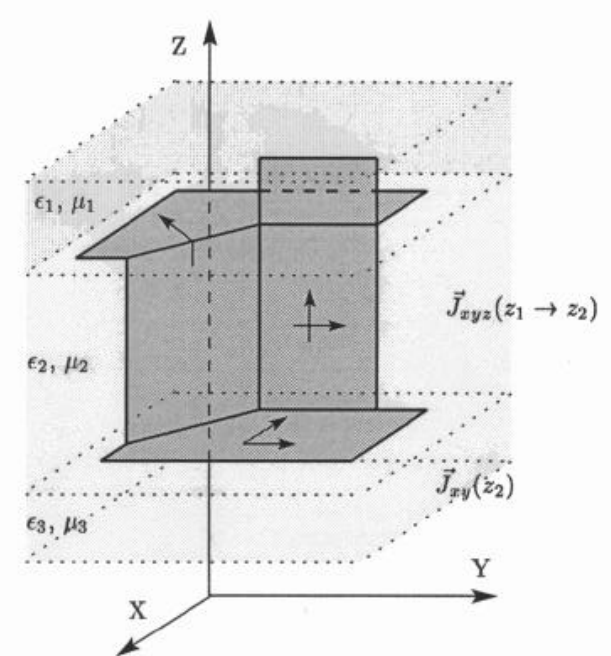

Figure 2: The 2.5D structure with full modeling of all current components on any cylindrical vertical current sheet.

The structures that can be modeled are depicted in Fig.2. Full rooftop modeling of all current components on vertical current sheets is performed, with the possibility to cut through an arbitrary number of dielectric layers. Vertical current sheets can also connect and intersect the horizontal sheets resulting in 'cornered' rooftop functions. This contrasts to a lot of commercially available (integral equation) field simulators which only have a very limited possibility for modeling vertical connections as shown in Fig.1. The limitations there are that only the z-component of the current is modeled, which makes it impossible to model extended vertical walls, and that the height of the vertical connection is small compared to the wavelength of the medium, such that the $\mathrm{z}$-current is also a constant. Often it is unclear whether the interfaces between dielectric layers can be crossed. Nevertheless, the term 2.5D or 3D planar is often already applied to the geometry of Fig.1. 


\section{THE ANALYTICAL INTEGRATIONS}

All field source relations involving a $z, z^{\prime}$-dependence are expressed in dyadic form and can be integrated in closed form. This is possible because the Green's functions in the spectral domain have a z, z' dependency corresponding to a transmission line problem. All integrals involving z, z' coordinates can therefore be performed analytically prior to the inverse Fourier transform. The remaining spatial integrals in the $x, y$-plane have to be done in a numerical manner after the inverse Fourier transform. For these integrals, a mixed potential representation retains its usual advantages. Expressed in a formula, the electromagnetic coupling is normally in the spatial domain computed as

$$
Z_{i j}=\iint_{S} \vec{E}_{S^{\prime}} \cdot \mathfrak{F}^{-1}\left\{G_{i j}\left(\tilde{k_{\rho}}, z, z^{\prime}\right)\right\} \vec{J}_{j} \mathrm{~d} S \mathrm{~d} S^{\prime}
$$

where the Green's functions is obtained via an inverse Fourier transform from the spectral domain. The basic idea is to perform all $z, z^{\prime}$ related integrals prior to the inverse Fourier transform,

$$
Z_{i j}=\iint_{l} \vec{E}_{l^{\prime}} \cdot \mathfrak{F}^{-1}\left\{\iint_{z} G_{z^{\prime}}\left(\tilde{k_{\rho}}, z, z^{\prime}\right) \mathrm{d} z \mathrm{~d} z^{\prime}\right\} \vec{J}_{j} \mathrm{~d} l \mathrm{~d} l^{\prime}
$$

because this can be done analytically. The inverse Fourier transform presents less problems with asymptotic/singular behaviour, since an integration has already been performed. The remaining numerical work are simple line integrals $l, l^{\prime}$ in the X, Y-plane. The process is depicted in Fig. 3. With this new approach a overall reduction of computation time and/or improved accuracy and convergence are envisaged.

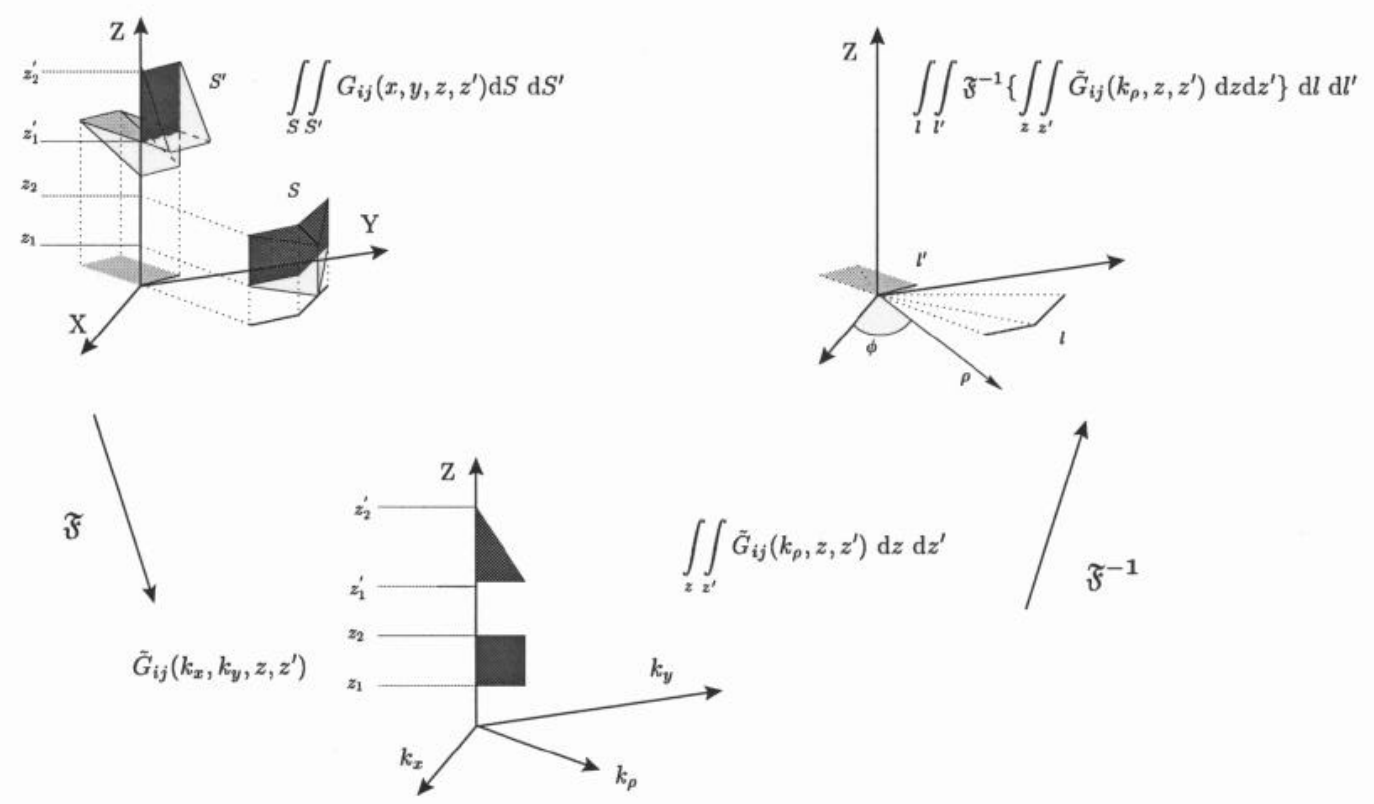

Figure 3: All integrations involving z, z' coordinates are done in closed form prior to the inverse Fourier transform. The remaining line integrals are done afterwards.

\section{EXAMPLES}

\section{IV.I. Full wave analysis of resonator of lumped elements}

Fig.4 shows a line above an infinite ground plane feeding a series connection of a multilayered capacitor and a square spiral inductor. The structures resides above an infinite ground plane in air. The dimensions of the lumped elements were chosen such that they behave indeed as discrete lumped elements below $1 \mathrm{GHz}$, while for higher frequencies their dimension is no longer negligible compared to the wavelength. For the lower frequencies an equivalent circuit model is sufficient to model the behaviour. Values for the static capacitance and quasi-static inductance can be obtained as Cstatic $=0.80 \mathrm{pF}, L_{\text {quasi-static }}=50 \mathrm{nH}$. Although the components themselves are still small below $1 \mathrm{GHz}$, it was already required for their series connection to insert a piece of transmission line in the circuit model of distance $d=18.125 \mathrm{~mm}$ to obtain the correct results. Fig.5 shows the amplitude of the $\mathrm{S}$ - parameters from $0.1-3.0 \mathrm{GHz}$, for the equivalent circuit model, and 3 full wave analyses. 


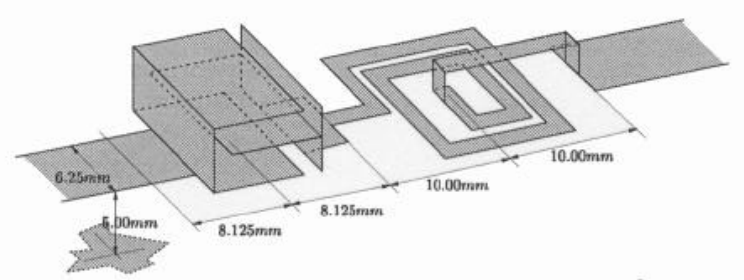

Figure 4: A series resonator composed of a multilayered capacitor and a square spiral inductor with airbridge.
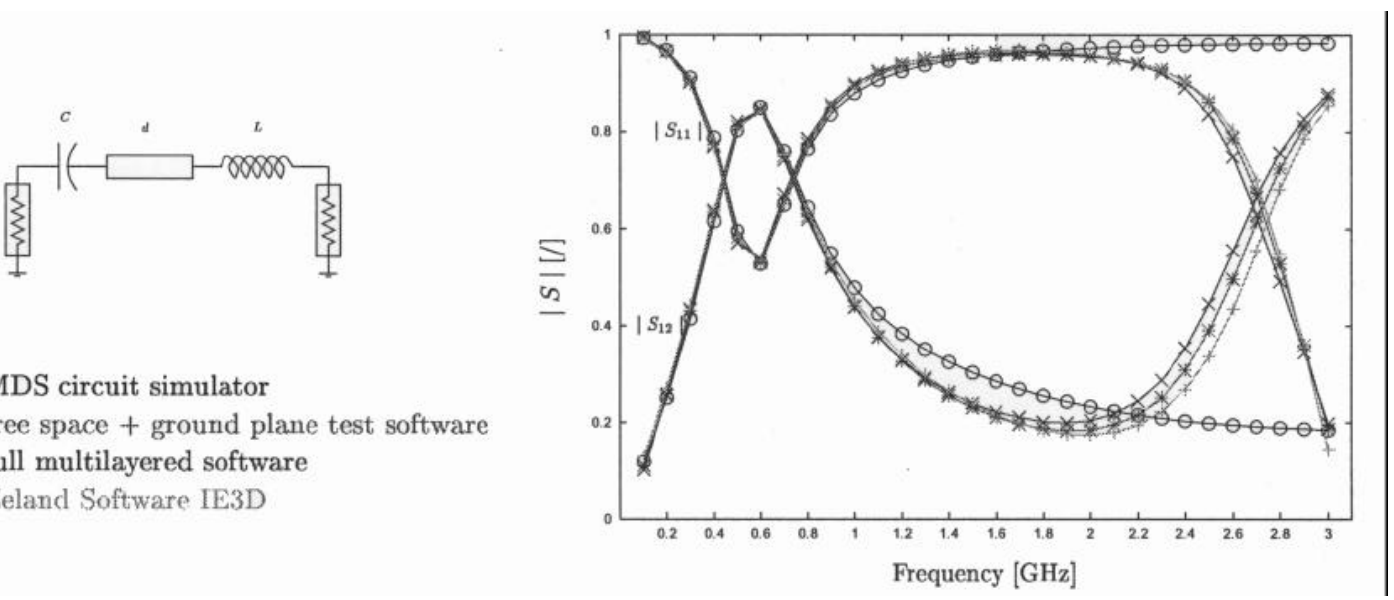

Figure 5: Comparison of the amplitude of $S_{11}, S_{12}$ for the series resonator of Figure 4 with a circuit simulator and 3 full wave simulations.

\section{IV.2. Patch antenna with vertical metalisation}

A two-level patch antenna with a vertical connecting piece is depicted in Fig. 6. The antenna consists of two patches, each at a different level of the two-layer medium, connected with a vertical metallic wall. The bottom layer has thickness $t_{1}=1.54 \mathrm{~mm}$ and a dielectric constant $\varepsilon_{r l}=3.00$. The top layer has thickness $t_{2}=1.57 \mathrm{~mm}$ and a dielectric constant $\varepsilon_{r 2}=2.33$. The other dimensions in Fig. 6 are $W_{p 1}=30 \mathrm{~mm}$, $L_{p}=40 \mathrm{~mm}, W_{s}=11.25 \mathrm{~mm}, L_{s}=5.0 \mathrm{~mm}$. The input impedance of this structure was measured by GayBalmaz and Mosig [2]. These measurements compare well with our computations as demonstrated in Fig. 7 which gives the response in the frequency range 1.9-2.3 GHz.

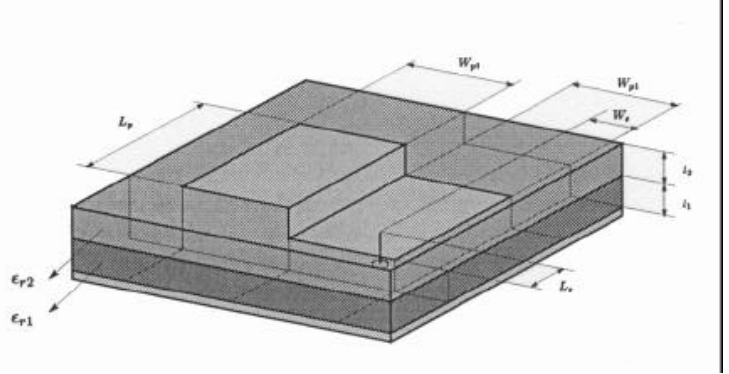

Figure 6: A two-level patch antenna with vertical metalisation in a two layer dielectric medium.

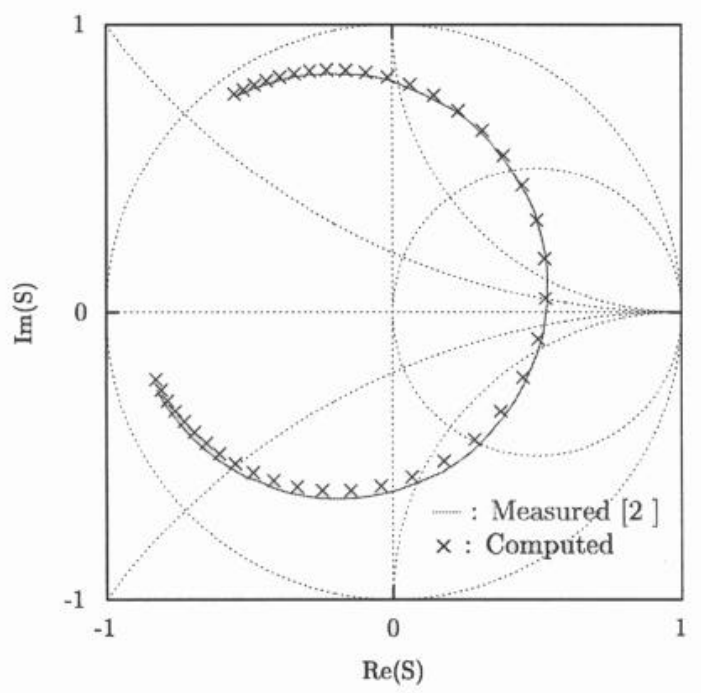

Figure 7: Input Impedance of the antenna of Fig. 6 from 1.9-2.3 GHz, 41 points. 


\section{IV.3. Full wave analysis of a microstrip rectangular inductor}

In the following example, we investigate the influence of the finite thickness of the upper piece of the airbridge of a microstrip rectangular spiral inductor. The example can also be found in Bunger and Arndt [1]. The main dimensions accompanying Fig. 8 are $d=635 \mu \mathrm{m}, w=625 \mu \mathrm{m}, s=312.5 \mu \mathrm{m}, \mathrm{h}=312.5 \mu \mathrm{m}, \boldsymbol{\varepsilon}_{r}=9.8$, and $t=0 \mu \mathrm{m}$. Our simulation results for the $S_{l 1}$, from $2-20 \mathrm{GHz}$ in 145 points are compared with those of Bunger and Arndt [1] for zero thickness $t=0 \mu \mathrm{m}$, and for finite thickness $t=312.5 \mu \mathrm{m}$.
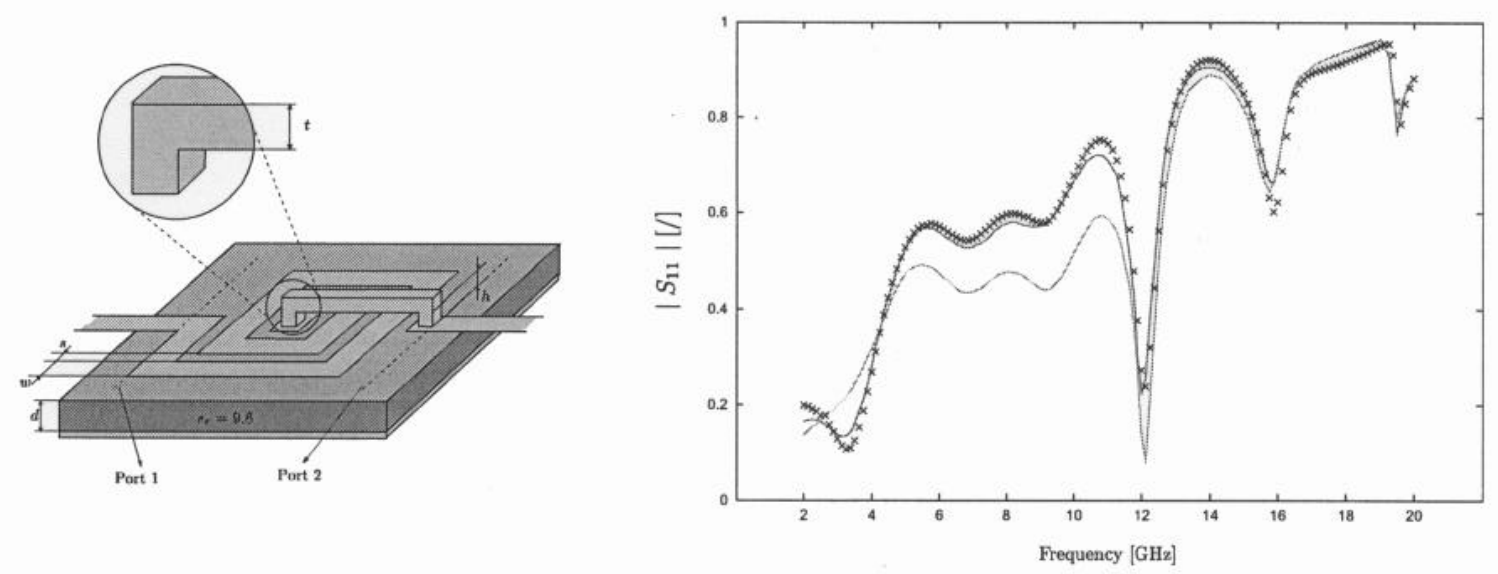

Influence thickness $t$ upper piece airbridge

$$
\begin{array}{rll}
t=000.0 \mu m & - & \text { R. Bunger, F. } \\
\text { Arndt [1] } & \text { R. Bunger, F. } \\
t=312.5 \mu t m & - & \begin{array}{l}
\text { Arndt [1] } \\
\text { full multilayered } \\
\text { software }
\end{array} \\
& \times
\end{array}
$$

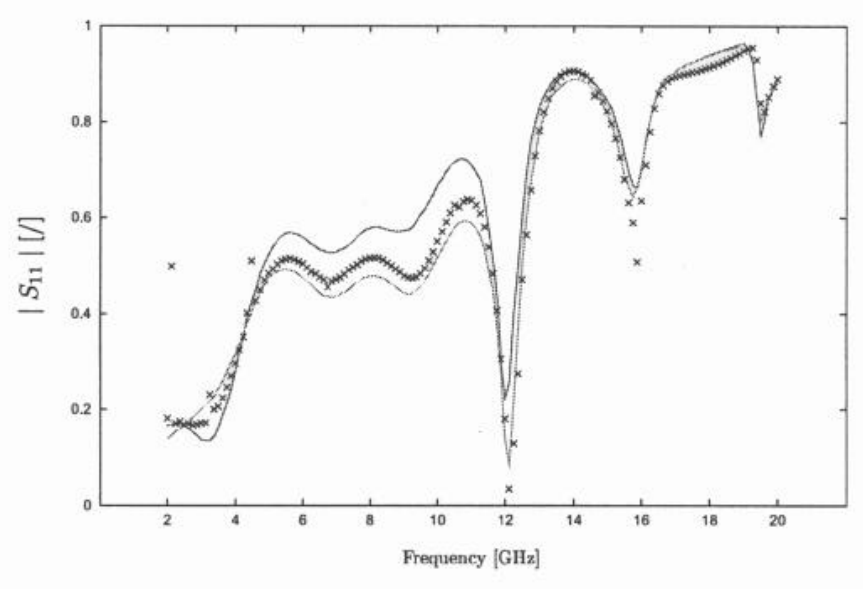

Figure 8: Influence of finite thickness of air bridge for a microstrip rectangular spiral inductor, $\left|S_{11}\right|$, from 2-20 GHz, 145 points.

\section{CONCLUSIONS}

It was demonstrated that the closed form expressions for the Green's functions in the spectral domain can be integrated analytically, prior to the inverse Fourier transform. Because of the analytical work, the method can be considered as a combination of a dyadic (analytical integrations in $z, z$ coordinates) and a mixed potential (numerical work in X, Y-plane) method. Examples show that this can even be done in an arbitrary multilayered medium.

\section{Ref e r e n e s}

[1] R. Bunger, and F. Arndt, "Efficient MPIE approach for the analysis of three-dimensional microstrip structures in layered media," IEEE Transactions on Microwave Theory and Techniques Vol. AP-45, No. 8, pp. 1141-1153, Aug. 1997.

[2] Ph. Gay-Balmaz, and J. R. Mosig, "Structures Rayonnantes 3-D planaires en milieu stratifié," Journeés Internationales de Nice sur les Antennes (JINA '96), pp. 127-130, 12-14 novembre 1996. 